\title{
Leaf Wand for Measuring Chlorophyll Fluorescence on Cylindrical Leaves and Its Application on Juncus roemerianus (Black Needlerush)
}

\author{
Patrick D. Biber \\ Department of Coastal Sciences, The University of Southern Mississippi, Ocean Springs, USA. \\ Email: patrick.biber@usm.edu
}

Received September $14^{\text {th }}, 2011$; revised October $25^{\text {th }}, 2011$; accepted November $15^{\text {th }}, 2011$

\begin{abstract}
Chlorophyll fluorescence is a well established technique to rapidly and non-invasively determine photosynthesis parameters in plant leaves. It can be used in both laboratory and field settings, and frequently dark-adaptation of a leaf sample is called for. In the field, this can be accomplished on flat leaves using standard leaf clips supplied by instrument manufacturers. However, not all plant leaves are flat, many are cylindrical or otherwise three-dimensional in shape. The standard leaf clip does not close fully on three-dimensional leaves, therefore, does not allow the sample to be properly dark adapted in the field. A new leaf "wand" was developed that can be slipped over an entire cylindrical leaf or culm of rushes and sedges for both light- and dark-adapted measurements. This new leaf wand is compared to the standard leaf clip (DLC-8) using a Walz mini-PAM on Juncus roemerianus (Black needlerush). Results indicate that darkadapted yield measurements are not significantly different between leaf clips, while light-adapted yields are higher with the leaf wand. The potential sources of difference in the optical path of the excitation light and fluorescence return are discussed and compared between leaf clips. Construction of specialized leaf wands should be considered for any leaves that are not flat and therefore do not fit the standard leaf clip for complete dark-adaptation under field conditions.
\end{abstract}

Keywords: Juncus roemerianus; Chlorophyll Fluorescence; Leaf Clip; Cylindrical Leaf; Optical Path Length

\section{Introduction}

Chlorophyll fluorescence is a tool to measure photophysiological processes in vivo and this technique has been used successfully to demonstrate physiological stress in a wide variety of plant species [1-4]. Various instruments have been designed and are commercially available to researchers [5], including portable instruments for field-application, such as the Walz Mini-PAM (Walz $\mathrm{GmBH}$ ). The availability of reliable instrumentation has led to chlorophyll fluorescence becoming a widely adopted, robust and reliable field technique that is easy to carry out, non-destructive, and rapid [6].

Chlorophyll fluorescence of photosystem II (PSII) can be measured by a variety of techniques including the pulse amplitude modulated (PAM) technique. Chlorophyll fluorescence can provide an instantaneous measure of the effective quantum yield $(\Delta \mathrm{F} / \mathrm{Fm}$ ') of PS II under prevailing ambient light conditions [7]. Alternatively, more standardized differences among leaves can also be determined by measuring the potential quantum yield (Fv/Fm) of dark-adapted samples. A healthy plant absorbs light from the sun, and directs a proportion of the solar energy absorbed into photosynthesis. Generally, the maximum possible proportion of the solar energy absorbed into photosynthesis is around $83 \%$, equivalent to a quantum yield of 0.830 [6]. As plants become stressed, reductions in the quantum yield of photosynthesis are evident, which can be used as a rapid screening technique [8].

Leaf samples can be dark adapted with flat leaf clips that are supplied with the instrument. These are attached to a flat plant leaf and serve to occlude a small area of the leaf. After a pre-determined period of time, a shutter built into the clip is manually opened, exposing the leaf area under the clip to very low intensity red light transmitted through fiber-optics in the case of the Walz PAM. The chlorophyll in the dark-adapted area of the leaf fluoresces and the initial fluorescence (Fo) is recorded. Upon illumination with a high intensity burst of saturating actinic light through the fiber-optics, the pigments associated with PSII become overwhelmed and the maximal fluorescence (Fm) is recorded. The difference between the maximal and initial fluorescence levels (Fm-Fo) is called the variable fluorescence (Fv) and from this the 
ratio $\mathrm{Fv} / \mathrm{Fm}$, or potential quantum yield, is calculated. In an analogous fashion the effective quantum yield $(\Delta \mathrm{F} /$ Fm') can be determined on flat leaf samples that are not dark-adapted and are exposed to ambient (sun) light.

Any decline in this ratio (either $\Delta \mathrm{F} / \mathrm{Fm}$ or $\mathrm{Fv} / \mathrm{Fm}$ ) indicates a reduction in the efficiency with which light is converted to photosynthetic product and subsequently, growth or reproductive output, and such a decline is often seen when a plant becomes stressed [9-11]. Therefore, by using a chlorophyll fluorescence instrument and the supplied leaf clips in the field, one can easily measure both $\Delta \mathrm{F} / \mathrm{Fm}$ ' and $\mathrm{Fv} / \mathrm{Fm}$, to determine whether the leaf is under some kind of stress.

However, not all plant leaves are flat. Some common examples of cylindrically- or other-shaped leaves are conifer needles, leaves and culms of rushes and sedges, and succulent plants [12]. The problem of using the standard flat leaf clip that comes with the PAM on these leaves is one of geometry. The standard leaf clip does not close fully on three-dimensional leaves, therefore, does not allow the sample to be properly dark adapted in field to get a measure of $\mathrm{Fv} / \mathrm{Fm}$.

One solution that has been adopted by plant researchers is to use multiple leaves to create a flat surface that is amenable to use in standard leaf clips. This is the approach used primarily with conifer needles because of their small diameter $[13,14]$. However, this approach yields a population average response, not a single leaf response, and so may not be desirable for certain comparative analyses. It also does not work well in large diameter cylindrical leaves like those of rushes. Research in our laboratory frequently focuses on the Black Needlerush, Juncus roemerianus Scheele, which is a common dominant species in saltmarshes of the northern Gulf of Mexico region. Because of the inability to use the flat leaf clips supplied with the Walz PAM to get darkadapted measurements in the field, a leaf "wand" for cylindrical-shaped leaves and culms of rushes and sedges was developed. The aim of this paper is to describe how to construct this leaf wand, and to compare its efficacy against that of the standard leaf clip supplied with the Walz PAM in obtaining light- and dark-adapted yield measurements.

\section{Materials and Methods}

An inexpensive prototype leaf "wand" for use on Juncus leaves was developed. Each wand is made from rigid plastic tubing with rubber shutters that rotate open and closed around circular holes (PAM measurement ports) drilled into the middle. Supplies necessary are $50 \mathrm{~cm}$ long $\times 5.5 \mathrm{~mm}$ inner diameter rigid plastic tubing (1/4" PVC sink faucet connector) and $9.5 \mathrm{~mm}$ inner diameter rubber hose (3/8" automotive fuel hose or compressed air line). Holes were carefully drilled into one side of the PVC with a $9.5 \mathrm{~mm}(3 / 8$ ") drill bit, just larger than the probe diameter $(8 \mathrm{~mm})$ on the Walz mini-PAM. Holes were spaced $15 \mathrm{~cm}$ apart, at standard heights to compare lower $(8 \mathrm{~cm})$, mid $(23 \mathrm{~cm})$, and upper $(38 \mathrm{~cm})$ portions of leaves on Juncus, although other spacing can be created as needed (Figure 1).

To create shutters over the holes for dark-adapting the leaf section, short sections of rubber hose cut into $5 \mathrm{~cm}$ lengths were used. These pieces of rubber also had a carefully drilled/cut $1 \mathrm{~cm}$ diameter hole in the middle of one side, to position the PAM probe in-line with the opening in the PVC pipe underneath. Shutters fit tightly around the wand, but loose enough to allow the rubber shutter to rotate open and shut for dark-adapted measurements. Some silicone stop-cock grease applied to the inside of the rubber tubing made it easier to rotate over the PVC tubing. Alignment marks were drawn on the

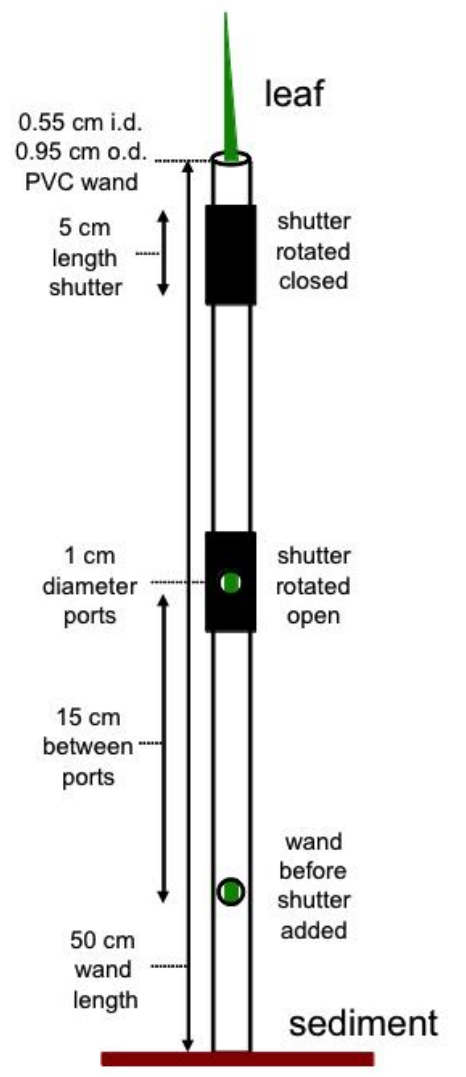

Figure 1. Diagram showing leaf wand construction, i.d. = inner diameter, o.d. = outer diameter. The leaf wand was slipped over the leaf targeted for PAM chlorophyll fluorescence measurements with readings taken at each measurement port. Light-adapted readings were taken directly after the wand was slipped over target leaf and dark-adapted readings were taken after shutters had been closed for at least 15 minutes. 
PVC pipe to indicate the top and bottom position of the shutter, as well as in line with the center of the measurement port to facilitate ease of positioning in field applications. The bottom of the wand sits flush with the sediment surface when the wand is slipped over the entire leaf (Figure 2).

For field measurements, multiple identical wands were constructed. Once a target leaf was selected, a wand was slipped over it and ambient light yield measurements were taken successively at each of the three PAM measurement ports on the wand, starting from the port closest to the sediment surface and proceeding up. At the completion of ambient light yield measurements for a specific leaf the shutters were rotated, closing off the measurement port to sunlight, and the sequence was repeated on the next leaf with a new wand. All leaves were given a minimum 15 minute dark incubation before rotating the shutter again to expose the measurement port for dark adapted yield measurements conducted following the same protocol as the ambient light yield measurements.

To test the null hypothesis of no mean difference by leaf clip type on chlorophyll fluorescence measurements, 10 pots with Juncus plants that had been grown from seed in the Mississippi Native Coastal Plants nursery greenhouse (http://www.usm.edu/gcrl/research/greenhouse. php) were randomly selected. At the time of the test, plants were 9 months of age and growing in $18 \mathrm{~cm}(6 ”)$ diameter plastic pots filled with potting soil, and subirrigated daily with fresh water. Plants had at least 3 leaves. Average ambient PAR was $195.5( \pm 12.04) \mathrm{mi}-$ cromols $/ \mathrm{m}^{2} / \mathrm{s}$ and air temperature was $27.5( \pm 0.0)$ degrees $\mathrm{C}$ at the time of the test measurements.

Fully green Juncus leaves $(\mathrm{n}=10)$ of similar developmental stage and size (Table 1) were compared used the standard Walz flat leaf clip (DLC-8) and the newly developed leaf "wand" (Figure 2). Leaf geometry affects the surface area of green tissue available to measure. Juncus leaves are cylindrical in shape, and taper from the base towards the tip, in the geometric shape of an elongated cone. Therefore, the diameter of the leaf decreases from the base to the tip. Meristematic growth of new tissue occurs at the base of the leaf near the sediment, so leaf tissue age increases with distance from the base. For these reasons, chlorophyll fluorescence was measured at three set distances along the leaf from the sediment up; these positions were $8 \mathrm{~cm}$ (bottom), $23 \mathrm{~cm}$ (mid) and

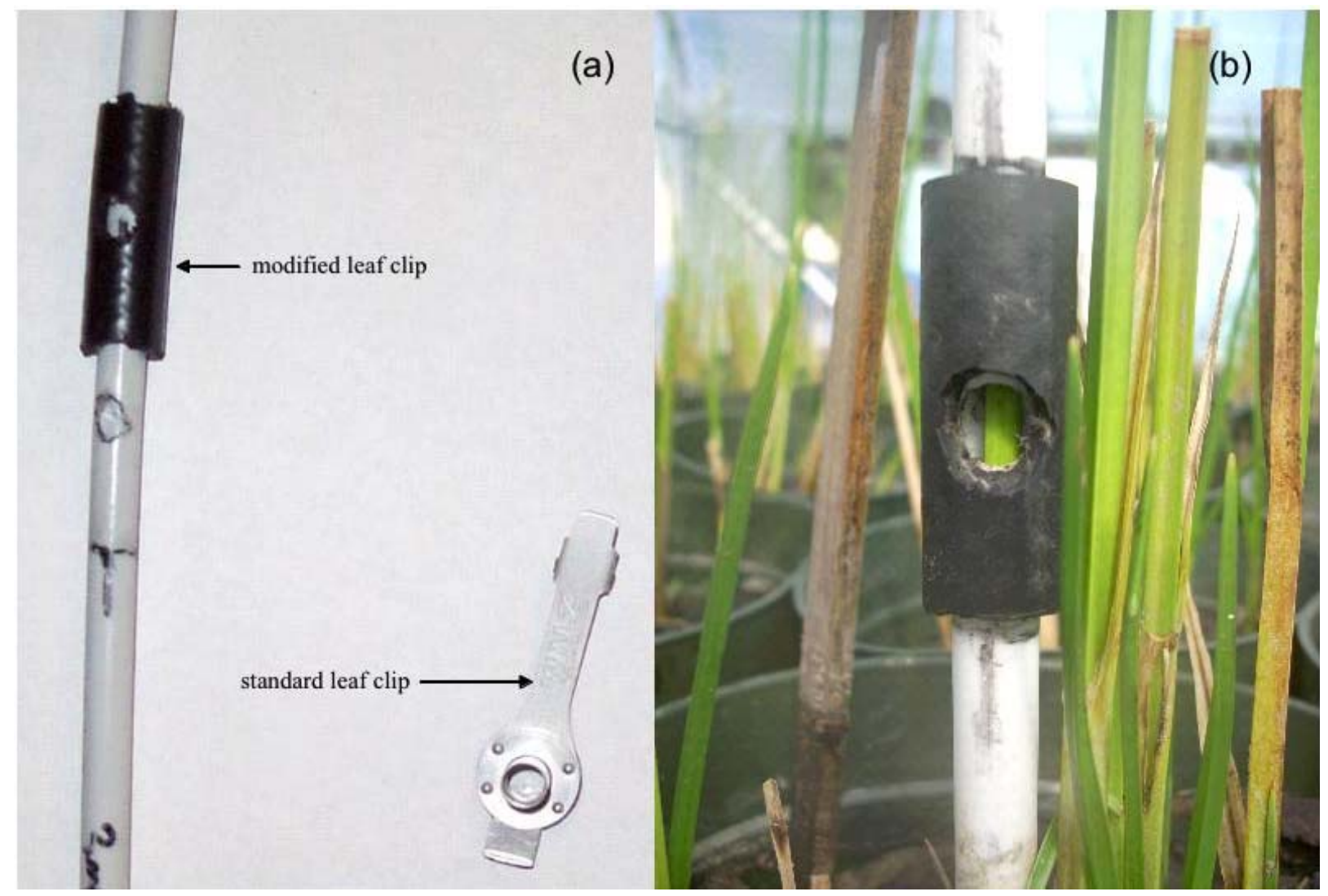

Figure 2. (a) Photograph of newly developed leaf wand with the rubber shutter located above the measurement port and instrument supplied Walz leaf clip (DLC-8), and (b) the leaf wand in action over a leaf showing the measurement port in the open position. 
Table 1. Mean (S.E.) of leaf measurements for 10 replicate Juncus leaves used to test a flat leaf clip and leaf wand. Positions are bottom $(B)=8 \mathrm{~cm}$, mid $(M)=23 \mathrm{~cm}$, top $(T)=38$ $\mathrm{cm}$ from base of leaf. Based on geometry, about half the circumference of the leaf is illuminated by the PAM probe tip, and this hemispherical surface area is expressed in equivalents of a flat leaf sample with an equal diameter of 7 $\mathrm{mm}\left(=0.385 \mathrm{~cm}^{2}\right)$. Top row shows Tukey's HSD indicated post-hoc groups for diameter, and the two derived quantities.

\begin{tabular}{cccc}
\hline \multirow{2}{*}{ Type } & \multicolumn{3}{c}{ Position along leaf } \\
\cline { 2 - 4 } & Bottom & Middle & Top \\
\hline CLIP & $\mathrm{A}$ & $\mathrm{AB}$ & $\mathrm{B}$ \\
Mean diameter (mm) & 1.758 & 1.632 & 1.506 \\
& $(0.0559)$ & $(0.0457)$ & $(0.0595)$ \\
Hemispherical S.A. $\left(\mathrm{mm}^{2}\right)$ & 19.320 & 17.936 & 16.551 \\
& $(0.6145)$ & $(0.5022)$ & $(0.6539)$ \\
Equivalent Flat Leaf Area & 0.502 & 0.466 & 0.430 \\
& $(0.0159)$ & $(0.0131)$ & $(0.0170)$ \\
WAND & $\mathrm{A}$ & $\mathrm{B}$ & $\mathrm{C}$ \\
Mean diameter (mm) & 1.716 & 1.481 & 1.207 \\
& $(0.0483)$ & $(0.0439)$ & $(0.0372)$ \\
Hemispherical S.A. (mm $\left.{ }^{2}\right)$ & 18.859 & 16.276 & 13.265 \\
& $(0.5304)$ & $(0.4820)$ & $(0.4089)$ \\
Equivalent Flat Leaf Area & 0.490 & 0.423 & 0.345 \\
& $(0.0138)$ & $(0.0125)$ & $(0.0106)$ \\
\hline
\end{tabular}

${ }^{\mathrm{a}}$ mean leaf length $=47.47 \pm(0.983) \mathrm{cm}$; ${ }^{\mathrm{b}}$ mean leaf length $=48.31 \pm(0.793)$ $\mathrm{cm}$.

$38 \mathrm{~cm}$ (top) (Table 1). Both light- $(\Delta \mathrm{F} / \mathrm{Fm}$ ') and darkadapted $(\mathrm{Fv} / \mathrm{Fm})$ measurements were made on the same leaf section with both leaf clip types.

Because repeated measurements on the same leaf section result in a reduction in fluorescence yield (nonphotochemical quenching) over time, different leaves for the leaf clip and leaf wand tests had to be used, so a paired t-test analysis was not appropriate. Instead, twoway analysis of variance on leaf clip type by position along the leaf, both as fixed factors, was performed on the light- and dark-adapted fluorescence data separately, after testing for homogeneity of variance and normality using MyStat 12.02 on a PC (http://www.systat.com). Data were log-transformed to meet the test assumptions. Post-hoc tests for means that were significantly different were performed using Tukey's Honestly Significant Difference (HSD) test. Box-plots of mean responses for base fluorescence (F, Fo), maximum fluorescence (Fm', Fm) and quantum yield $(\Delta \mathrm{F} / \mathrm{Fm}$ ', $\mathrm{Fv} / \mathrm{Fm})$ by leaf clip type and leaf position were created to aid in visualizing the results using R version 2.12.2 on Mac OS X (www.r-project. org).

\section{Results}

There was no significant difference in length of leaves tested between leaf clip and leaf wands $(\mathrm{p}=0.2374$, Table 1). The mean diameter of the leaves decreased significantly from the base to the tip ( $p=0.0103$ clip; $p=$ 0.0001 wand). In addition to diameter, leaf surface area interrogated by the PAM probe was calculated, based on the assumption that half the surface area of the leaf was exposed to the actinic light pulse and contributed to the fluorescence signal returned. The formula used was $(2 \times$ $\mathrm{Pi} \times 0.5 \mathrm{dia}) / 2 \times \mathrm{h}$, where $\mathrm{h}$ was $7 \mathrm{~mm}$, i.e., the diameter of the mini-PAM fiber-optic. From this hemispherical surface area, the equivalent surface area was determined compared to a flat leaf also having a diameter of $7 \mathrm{~mm}$ $\left(38.47 \mathrm{~mm}^{2}\right.$ ) exposed to interrogation and detection by the mini-PAM (Table 1).

Boxplots of light-adapted and dark-adapted leaves indicate that data collected using the standard flat leaf clip (DLC-8) had both lower mean values and less variance than data collected with the leaf wand for base (F, Fo), maximum (Fm', Fm), and variable $(\Delta \mathrm{F}, \mathrm{Fv})$ fluorescence (Figures 3 and 4). However, this pattern did not extend to the yield values, where the leaf wand had equal or lower variability than the flat leaf clip (Figures 3(d) and 4(d)), and similar means for dark-adapted samples between leaf clip types (Figure 4(d)). In contrast, the leaf wand had higher means for light-adapted yields (Figure 3(d)) when compared to the flat leaf clip.

Results of the two-way ANOVA on leaf clip versus leaf wand by position (bottom, middle, top) of the leaf tested are shown in Table 2. For light-adapted leaves measured immediately after placement of the flat clip or wand, there were significant differences between clip types and position along the leaf for all three metrics (F, Fm', and effective quantum yield). However, there was no significant interaction effect of clip $\times$ position for any

Table 2. Results of two-way ANOVA on three metrics derived from chlorophyll fluorescence on Juncus leaves (n = 10) using two leaf clips (flat leaf clip vs leaf wand) at three positions along the length of the leaf $(8,23,38 \mathrm{~cm}$ from base). Data were log-transformed, and yield was $\log (X+1)$ transformed prior to analysis to satisfy assumptions of normality and homoscedasticity. P-values are considered significant at an alpha-level $=\mathbf{0 . 0 5}$.

\begin{tabular}{cccc}
\hline \multirow{2}{*}{ p value } & \multicolumn{3}{c}{ Chlorophyll Fluorescence Parameter } \\
\cline { 2 - 4 } & F/Fo & Fm'/Fm & Yield \\
\hline Light adapted & & \\
Clip (C) & 0.000 & 0.000 & 0.000 \\
Posn (P) & 0.000 & 0.000 & 0.049 \\
C $\times$ P & 0.698 & 0.793 & 0.600 \\
Dark adapted & & \\
Clip (C) & 0.000 & 0.000 & 0.164 \\
Posn (P) & 0.001 & 0.000 & 0.001 \\
C $\times \mathbf{P}$ & 0.163 & 0.239 & 0.690 \\
\hline
\end{tabular}




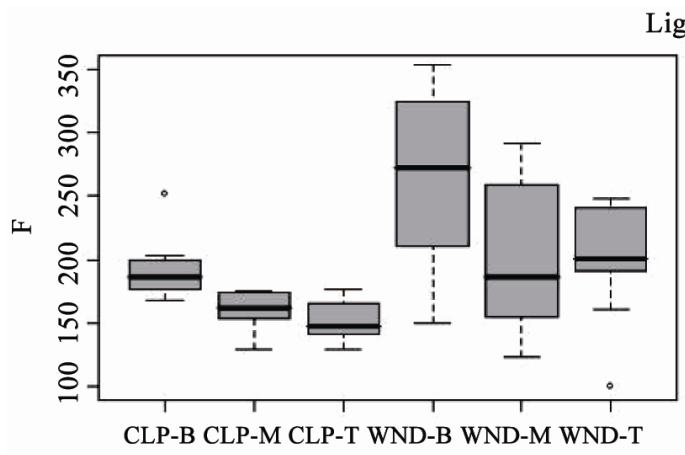

(a)

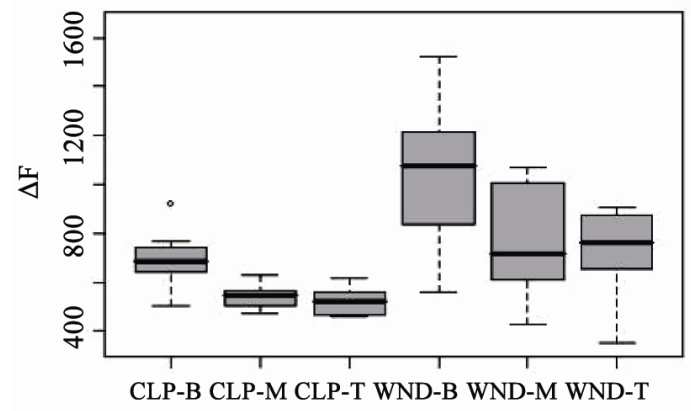

(c)

Light-adapted

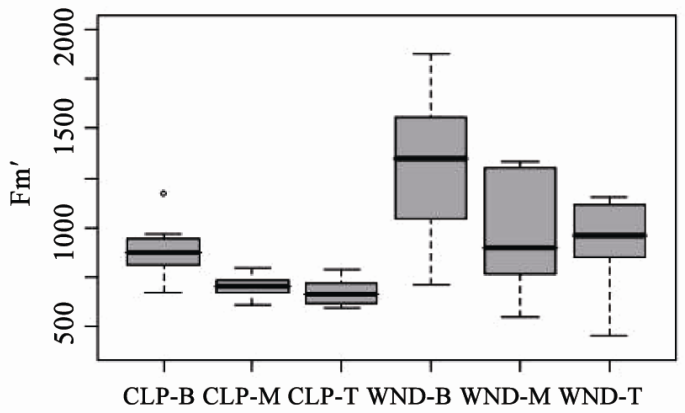

(b)

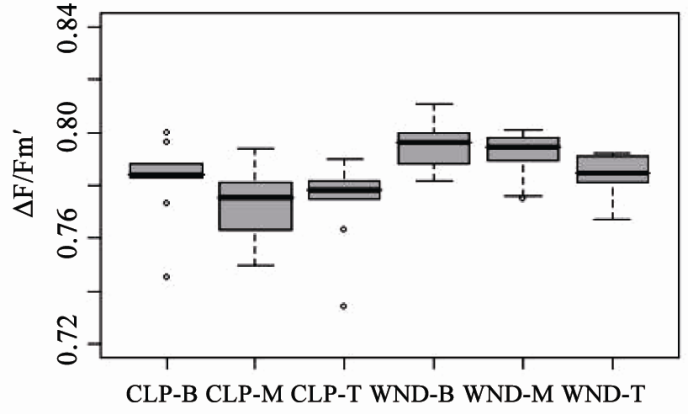

(d)

Figure 3. Boxplots of light-adapted chlorophyll fluorescence parameters for leaf clip and leaf wand treatments from $\mathbf{n}=10$ Juncus leaves measured at three different heights $(B=8, M=23, T=38 \mathrm{~cm}$ from sediment). Panels are (a) base fluorescence (F), (b) maximum fluorescence (Fm'), (c) variable fluorescence ( $\Delta F)$, and (d) Effective Quantum Yield ( $\Delta F / F m$ '), from flat leaf clips (CLP) and leaf wand (WND).

of the three metrics (Table 2). Tukey's post-hoc test indicates that measurement values using the leaf wand were always significantly greater than those obtained with the standard flat leaf clip. For dark-adapted leaves (minimum 15 minutes after the flat clip shutter or the wand shutter was rotated closed) there were significant differences between clips for Fo, Fm, but not potential quantum yield (Table 2). In all three metrics, there were significant differences in the position along the leaf. As for the light measurements, there was no significant interaction effect of clip $\times$ position (Table 2). Tukey's post-hoc test indicates that measurement values using the leaf wand were significantly greater than those obtained with the standard flat leaf clip for Fo and Fm, but not for Fv/Fm data.

These results suggest that there are differences between leaf clip types, as is to be expected given the different optical environment created in the cylindrical leaf wand compared to the flat leaf clip (Figure 5). Darkadapting the cylindrical leaves with the leaf wand resulted in slightly higher yield (not significant), evidenced by the significantly higher values of the base and maximum fluorescence values compared to the flat leaf clip. The variability in measurements also tended to be higher with the leaf wand than the standard leaf clip for both light- and dark-adapted leaf samples.

\section{Discussion}

The leaf wand has been used in over 5000 field and laboratory measurements on Juncus (rush) and Schoenoplectus (sedge) spp. to collect light- and dark-adapted measurements on these cylindrically shaped leaves and culms. Occasionally there is a problem with a small diameter leaf not aligning with the centerline of the hole, resulting in a low Fo error by the PAM instrument (Fo < 130 ), but it can be corrected by repositioning the wand over the leaf. For very thin leaves in immature individuals, it may be necessary to use multiple leaves within the same wand to "fill-in" the probe window. This is undesirable for comparison to other data as it is a sample average and not an individual measurement. Nonetheless, this approach has become a commonly used technique for conifer needles that are individually too narrow to give a good signal response for many optical measurements $[13,14]$.

Juncus and other plants with leaves that are not flat may pose problems for accurate fluorescence measurement. The issue with cylindrical leaves is the potential scattering of the light signal away from the detector due to the curved leaf surface, resulting in erroneous readings 


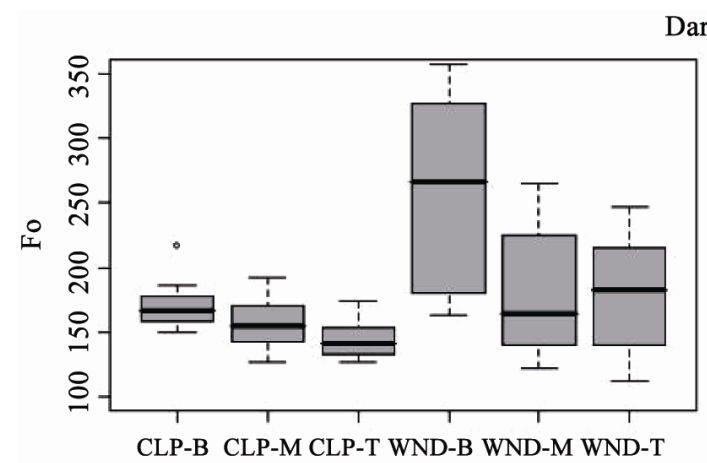

(a)

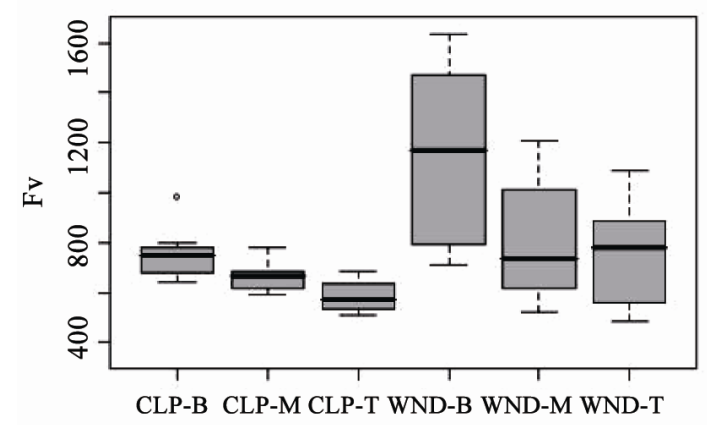

(c)

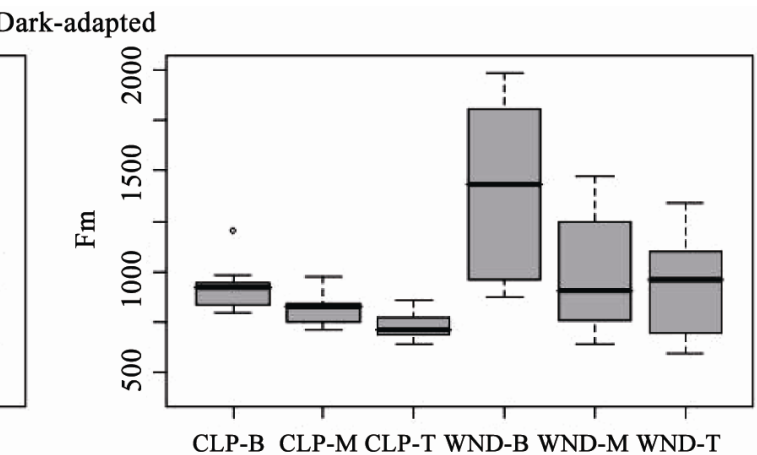

(b)

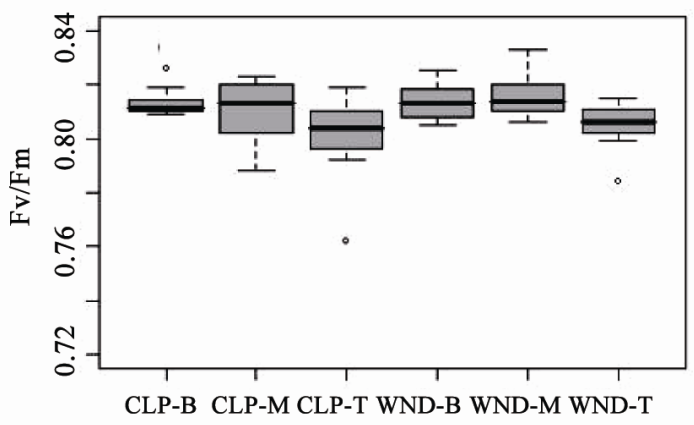

(d)

Figure 4. Boxplots of dark-adapted chlorophyll fluorescence parameters for leaf clip and leaf wand treatments from $\mathbf{n}=10$ Juncus leaves measured at three different heights $(B=8, M=23, T=38 \mathrm{~cm}$ from sediment). Panels are (a) base fluorescence (Fo), (b) maximum fluorescence (Fm), (c) variable fluorescence (Fv), and (d) Potential Quantum Yield (Fv/Fm), from flat leaf clips (CLP) and leaf wand (WND).

[15]. Some of these problems can be overcome by standardizing leaf diameter, leaf age, and position along the leaf to determine relative differences within the same species [16]. However, problems exist when trying to compare between species with different leaf morphologies $[14,17]$.

If a plant leaf was a perfect optical medium, and only chlorophyll attenuated the measuring light beam, then fluorescence values would be proportional to chlorophyll pigment concentration [18-20]. Unfortunately, leaves are not perfect optical systems because light is refracted as well as focused by the epidermal cells resulting in variation in the light intensity at the chloroplast. Columnar palisade cells facilitate the penetration of collimated light into the leaf interior where, if it is not absorbed, it is scattered by the spongy mesophyll, which increases the path length, and helps increase the probability of light absorption [21]. Chlorophyll containing palisade tissues are radially arranged around the perimeter of Juncus leaves, with the interior composed of unpigmented parenchyma cells [22]. Further, chlorophyll pigments are packaged in the chloroplasts, resulting in differential wavelength-specific light extinction inside the leaf (invivo) compared to measurements on extracted pigments (in-vitro). Reference [21] found a lower extinction coef- ficient at $450 \mathrm{~nm}$ (blue light) in spinach leaves compared to that of extracted pigments, which they attribute to the sieve effect, where packaging of chlorophyll within the chloroplast enhances the efficiency of light penetration at wavelengths that are strongly absorbed, i.e. red and blue light. By contrast, they found greater extinction at 550 $\mathrm{nm}$ (green light) when measured in-vivo compared with that of extracts in-vitro, which reflects light scattering to increase the effective path length and increases the probability of absorption by chlorophyll at weakly absorbed wavelengths [21,23].

An implicit fundamental assumption of the measurement of the quantum yield ( $\Delta \mathrm{F} / \mathrm{Fm}$ or $\mathrm{Fv} / \mathrm{Fm})$ is that all cells contributing to the measured fluorescence signal are 1) equally exposed to the measured level of actinic light, 2) exposed to light pulses which are effectively saturating and 3) equally exposed to measuring light and emitting equally detectable fluorescence [11,24]. As [24] elegantly points out, light attenuation within optically dense samples follows approximately Beer-Lambert dynamics, which greatly complicates the fluorescence signal returned to the detector (Figure 5). Furthermore, light transmission, scattering, absorbance, and reflectance are all strongly affected by turgor pressure, such that leaf tissue at the time of measurement may function more or less as 


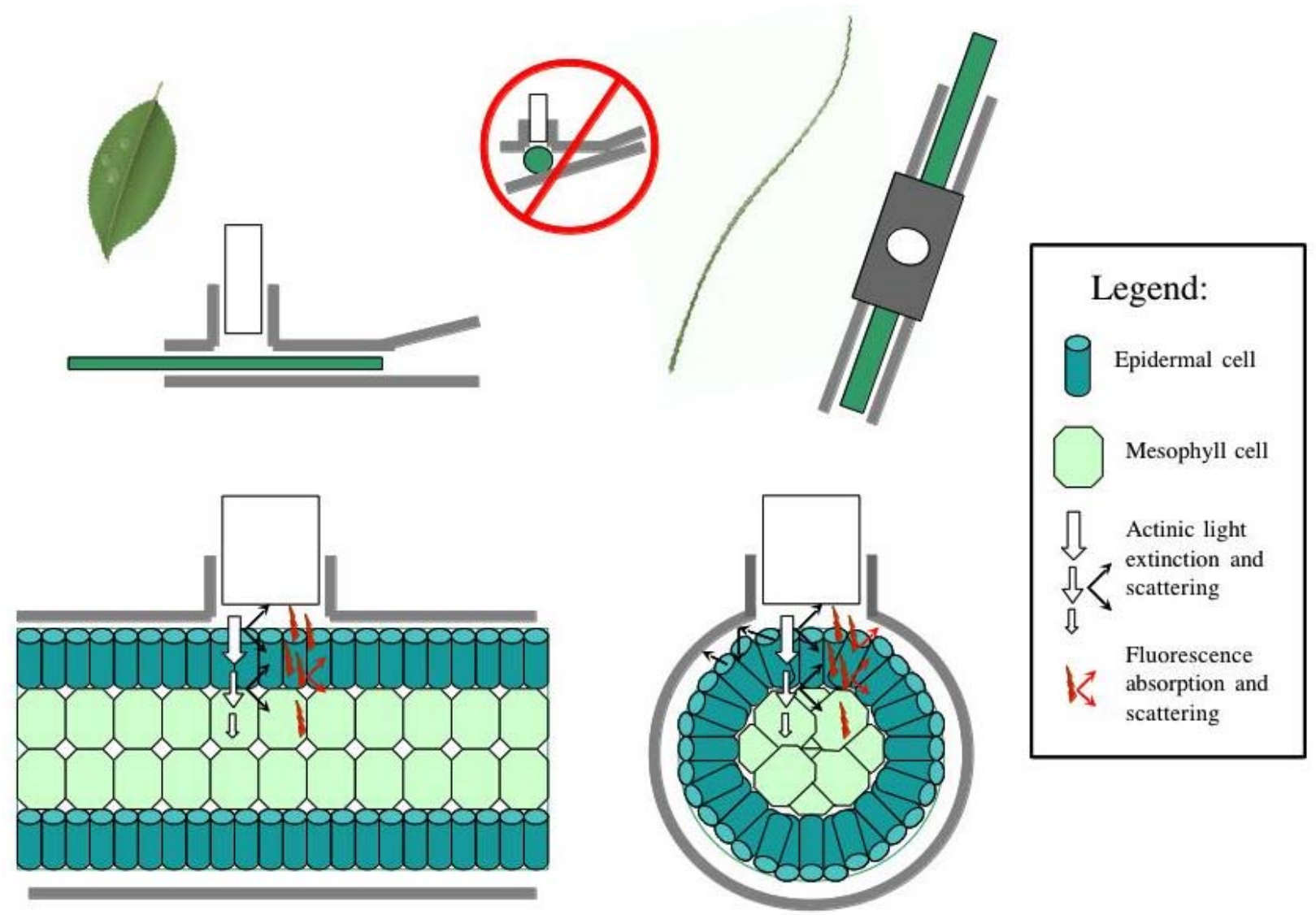

Figure 5. Diagram of changes in optical properties between the flat leaf clip and the cylindrical leaf wand, with light scattering and fluorescence scattering indicated. Cylindrical leaves are more prone to sub-optimal light path issues, reducing detection efficacy at the PAM sensor. Legend indicates symbol properties.

a collimating lens for incident irradiance [20,21]. For these reasons the measurements used to derive the yield ratio can be substantially influenced by leaf optical properties as well as environmental (e.g., light intensity) and physiological (e.g., turgor) parameters [5,6].

Cylindrical leaves are more likely than flat leaves to have reflection and refraction of irradiated actinic light at the leaf-air interface lead to some loss of signal from and to the PAM probe window (Figure 5). Fluorescence will also have similar problems with some loss of signal due to light paths that lead away from the detector window. These optical problems not-with-standing, the leaf wand does guarantee complete dark-adaptation of the entire leaf, which is not the case with the standard leaf clip where, depending on leaf diameter, substantial light leakage can occur resulting in an improperly dark-adapted sample (Figure 5). Using the leaf wand for both lightand dark-adapted measurements on the same sample, will result in an equal range of variability due to the above described optical constraints, and is a better solution than mixing leaf clip types, i.e. compare variance due to clip types in Figures $\mathbf{3}$ and $\mathbf{4}$. In this study significant leaf clip effects were found using a population of Juncus leaves measured with both clip types, indicating that optical arrangement of the PAM probe in relation to the leaf sample was significantly affected by the type of leaf clip used. In general, leaf diameter could be used as a visual predictor of a good fluorescence measurement, with thin leaves that occupy less than half the optical port more likely to be out of optimal alignment during a measurement. These data-points can later be easily identified in the downloaded dataset by sorting the data in ascending order of Fo. Values less than 130 RFU (relative fluorescence units) are indicated at the time of collection by an audible alarm. Values less than 100 RFU are generally associated with poor Fm and yield ratio values compared to larger diameter samples, and should be discarded from subsequent analyses.

\section{Conclusions}

Based on the experience with determining quantum yields on the cylindrical leaves of Juncus roemerianus, a dominant salt-marsh species in the northern Gulf of 
Mexico, it is clear that further development and testing of non-standard leaf clips for use on various 3-dimensional leaf geometries is warranted for specialty field-applications on certain species. Any newly developed leaf clips will need to be tested explicitly by comparing them with the standard leaf clip as optical properties are likely to be significantly affected. A further test of the efficacy of different leaf clip types could be done by comparing results obtained with those from an Imaging PAM (www. walz.com/products/chl_p700/imaging-pam_ms/introducti on.html), as this instrument does not suffer the same geometrical constraints, but is less field-portable for darkadapted samples. In this study, an experimental prototype leaf wand was found to have higher variability than the standard flat leaf clip in measurements of chlorophyll fluorescence parameters, but this was cancelled out in the calculation of the yield ratio. The leaf wand allowed complete dark-adaptation of the full leaf under field conditions, which would not have been possible using the flat leaf clip.

\section{REFERENCES}

[1] C. Critchley and R. M. Smilie, "Leaf Chlorophyll Fluorescence as an Indicator of Photoinhibition in Cucumis sativus L.," Australian Journal of Plant Physiology, Vol. 8, No. 2, 1981, pp. 133-141. doi:10.1071/PP9810133

[2] M. Havaux and R. Lannoye, "Chlorophyll Fluorescence Induction: A Sensitive Indicator of Water Stress in Maize Plants,” Irrigation Science, Vol. 4, 1983, pp. 147-151.

[3] J. R. Bowyer, P. Camilleri and W. F. J. Vermaas, "Photosystem II and Its Interaction with Herbicides,” In: N. R. Baker and M. P. Percival, Eds., Herbicides, Topics in Photosynthesis, Elsevier, Amsterdam, 1991, pp. 27-85.

[4] D. L. Filiault and J. C. Stier, "The Use of Chlorophyll Fluorescence in Assessing the Cold Tolerance of Three Turfgrass Species," Wisconsin Turfgrass Research, Vol. 16, 1999, pp. 109-110.

[5] H. R. Nordenkampf, S. P. Long, N. R. Baker, G. Oquist, U. Schreiber and E. G. Lechner, "Chlorophyll Fluorescence as a Probe of the Photosynthetic Competence of Leaves in the Field: A Review of Current Instrumentation,” Functional Ecology, Vol. 3, No. 4, 1989, pp. 497514. doi:10.2307/2389624

[6] K. Maxwell and G. N. Johnson, "Chlorophyll Fluorescence: A Practical Guide,” Journal of Experimental Botany, Vol. 51, No. 345, 2000, pp. 659-668. doi:10.1093/jexbot/51.345.659

[7] B. Genty, J. Briantais and N. Baker, “The Relationship between the Quantum Yield of Photosynthetic Electron Transport and Quenching of Chlorophyll Fluorescence,” Biochemica et Biophysica Acta, Vol. 990, No. 1, 1989, pp. 87-92. doi:10.1016/S0304-4165(89)80016-9

[8] U. Schreiber, H. Horman, C. Neubauer and C. Klughammer, "Assessment of Photosystem II Photochemical
Quantum Yield by Chlorophyll Fluorescence Quenching Analysis," Australian Journal of Plant Physiology, Vol. 22, No. 2, 1995, pp. 209-220. doi:10.1071/PP9950209

[9] E. D. Schulze and M. M. Caldwell, "Ecophysiology of Photosynthesis,” Springer, Berlin, 1990.

[10] G. H. Krause and E. Weis, "Chlorophyll Fluoresence and Photosynthesis: The Basics," Annual Review of Plant Physiology and Plant Molecular Biology, Vol. 42, 1991, pp. 313-349. doi:10.1146/annurev.pp.42.060191.001525

[11] K. Rohacek and M. Bartak, "Technique of the Modulated Chlorophyll Fluorescence: Basic Concepts, Useful Parameters, and Some Applications,” Photosynthetica, Vol. 37, No. 3, 1999, pp. 339-363. doi:10.1023/A:1007172424619

[12] R. K. Godfrey and J. W. Wooten, "Aquatic and Wetlands Plants of the Southeastern United States," University of Georgia Press, Athens, 1979.

[13] A. D. Richardson, G. P. Berlyn and T. G. Gregoire, "Spectral Reflectance of Picea rubens (Pinaceae) and Abies balsamea (Pinaceae) Needles along an Elevational Gradient, Mt. Moosilauke, New Hampshire, USA,” American Journal of Botany, Vol. 88, No. 4, 2001, pp. 667676. doi: $10.2307 / 2657067$

[14] T. Brodribb and R. S. Hill, "Light Response Characteristics of a Morphologically Diverse Group of Southern Hemisphere Conifers as Measured by Chlorophyll Fluorescence,” Oecologia, Vol. 110, No. 1, 1997, pp. 10-17. doi:10.1007/s004420050127

[15] P. D. Biber, "Evaluating a Chlorophyll Content Meter on Three Coastal Wetland Plant Species,” Journal of Agricultural, Food, and Environmental Sciences, Vol. 1, No. 2, 2007, pp. 1-11.

[16] G. Naidoo and J. Kift, "Responses of the Saltmarsh Rush Juncus Kraussii to Salinity and Waterlogging," Aquatic Botany, Vol. 84, No. 3, 2006, pp. 217-225. doi:10.1016/j.aquabot.2005.10.002

[17] K. L. Castro-Esau, G. A. Sanchez-Azofeifa, B. Rivard, S. J. Wright and M. Quesada, "Variability in Leaf Optical Properties of Mesoamerican Trees and the Potential for Species Classification,” American Journal of Botany, Vol. 93, No. 4, 2006, pp. 517-530. doi:10.3732/ajb.93.4.517

[18] A. D. Richardson, S. P. Duigan and G. P. Berlyn, “An Evaluation of Noninvasive Methods to Estimate Foliar Chlorophyll Content," New Phytologist, Vol. 153, No. 1, 2002, pp. 185-194. doi:10.1046/j.0028-646X.2001.00289.X

[19] J. Markwell, J. Osterman and J. Mitchell, "Calibration of the Minolta SPAD-502 Leaf Chlorophyll Meter," Photosynthesis Research, Vol. 46, No. 3, 1995, pp. 467-472. doi:10.1007/BF00032301

[20] M. N. Merzlyak, O. B. Chivkunova, T. V. Zhigalova and K. R. Naqvi, "Light Absorption by Isolated Chloroplants and Leaves: Effect of Scattering and Packaging," Photosynthesis Research, Vol. 102, No. 1, 2009, pp. 31-41. doi:10.1007/s11120-009-9481-8

[21] T. C. Vogelmann and J. R. Evans, "Profiles of Light Absorption and Chlorophyll within Spinach Leaves from 

on Juncus roemerianus (Black Needlerush)

Chlorophyll Fluorescence,” Plant, Cell and Environment, Vol. 25, No. 10, 2002, pp. 1313-1323. doi:10.1046/j.1365-3040.2002.00910.x

[22] L. Eleuterius, "Vegetative Morphology and Anatomy of the Salt Marsh Rush, Juncus roemerianus," Gulf Research Reports, Vol. 5, No. 2, 1976, pp. 1-10.

[23] D. W. Lee, S. F. Oberbauer, P. Johnson, B. Krishnapilay, M. Mansor, H. Mohamad and S. K. Yap, "Effects of Irradiance and Spectral Quality on Leaf Structure and
Function in Seedlings of Two Southeast Asian Hopea (Dipterocarpaceae) Species," American Journal of Botany, Vol. 87, No. 4, 2000, pp. 447-455. doi:10.2307/2656588

[24] J. Serodio, “Analysis of Variable Chlorophyll Fluorescence in Microphytobenthos Assemblages: Implications of the Use of Depth-Integrated Measurements,” Aquatic Microbial Ecology, Vol. 36, No. 2, 2004, pp. 137-152. doi:10.3354/ame036137 